\title{
Enhanced field-of-view high- resolution lattice light sheet microscopy with phased array beam formers
}

J. Witzens, M. Spehr, P. Van Dorpe, N. Verellen

J. Witzens, M. Spehr, P. Van Dorpe, N. Verellen, "Enhanced field-of-view highresolution lattice light sheet microscopy with phased array beam formers," Proc. SPIE 11652, Adaptive Optics and Wavefront Control for Biological Systems VII, 1165207 (5 March 2021); doi: 10.1117/12.2576700 


\title{
Enhanced field-of-view high-resolution lattice light sheet microcopy with phased array beam formers
}

\author{
J. Witzens*a, M. Spehr ${ }^{\mathrm{b}}$, P. Van Dorpe ${ }^{\mathrm{c}}$, N. Verellen ${ }^{\mathrm{c}}$

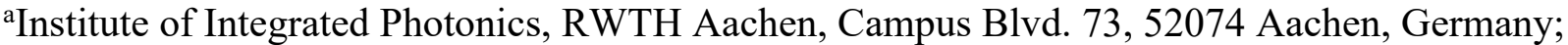 \\ ${ }^{b}$ Chemosensation Laboratory, Institute of Biology II, RWTH Aachen, Worringerweg 3, 52074 \\ Aachen, Germany; \\ cimec, Kapeldreef 75, 3001 Leuven, Belgium
}

\begin{abstract}
The data acquisition speed of point scanning microscopy techniques at sub-cellular resolution limits imaging of large samples, as sample stability and focus drift are becoming an issue. Therefore, light sheet fluorescent microscopy (LSFM) has become the method of choice for imaging cleared samples. However, this method still suffers from a trade-off between imaging depth and resolution, due to diffraction of the illuminating beam limiting the achievable field of view. After improvement of the latter with non-diffracting Bessel beams, lattice light sheet microscopy has further reduced the imaging point-spread-function (PSF) as well as substantially reduced illumination intensities and the resulting phototoxicity by enabling illumination of entire light sheets over large lateral extents. Here, we propose further improvement by generation of structured light sheets via phased arrays implemented as silicon nitride photonic integrated circuits (PICs). Beam generation with PICs results in much higher power efficiency than beam forming with conventional liquid crystal based spatial phase modulators, as it does not require the use of narrow blocking apertures. Moreover, this approach enables increased control over the generated field profile. Modeling of concrete PIC concepts indicates that sub-cellular resolution with mm scale imaging depths can be concomitantly achieved. Maintaining a small PSF across the entire light sheet, along the axis perpendicular to the direction of light propagation, is sacrificed in order to maintain it over an increased imaging depth along the beam propagation axis. Rapid lateral scanning of the illumination beam inside the plane of the light sheet is then obtained by scanning of the laser wavelength within the excitation spectrum of the target fluorescent protein, allowing for a wide bidirectional field-of-view with high resolution.
\end{abstract}

Keywords: lattice light sheet microscopy, structured illumination, phased arrays, beam forming, silicon nitride, photonic integrated circuits.

\section{INTRODUCTION}

Developing imaging methods that allow $(i)$ simultaneous activity measurements of up to 100,000 neurons in large fields of view, ${ }^{1,2}$ and/or (ii) large-scale neuroanatomical analysis of network connectivity in intact brains at high spatial resolution, is the technological basis for future advances in understanding the brain and developing effective treatment for neurological and psychiatric disorders. LSFM - declared as "Method of the Year 2014" by Nature Methods ${ }^{3}$ - holds great promise to meet these challenging demands.

The inherent three-dimensional (3D) structure of cells and tissues is usually a critical determinant of cell and organ functionality. This is particularly true for the central nervous system, ${ }^{4}$ in which the intricate complex morphologies of neurons and their long-range dendritic and axonal projections provide the structural foundation for fast information processing within and between neural circuits. Moreover, detailed insight into neurodevelopmental processes - and, consequently, into developmental abnormalities that frequently underlie neuro-pathologies - usually emerges from 3D structural information. Accordingly, there is increasing demand in (neuro)science for volumetric imaging techniques able to visualize the relevant morphological/anatomical structures in their intact environment with high spatial precision. In the past, the method of choice to reconstruct 3D information put into register a series of serial thin sections. However, this cumbersome approach is technically challenging due to loss or distortion of individual sections that become torn, folded, compressed, or stretched. ${ }^{4}$ Therefore, volumetric reconstructions were mostly limited to smaller samples and, frequently, results turned out unsatisfactory. At the ultrastructural scale, serial block-face scanning electron microscopy ${ }^{5}$ has recently become the gold standard to visualize (sub)cellular structures with extreme accuracy. This method, however, is limited by tedious data acquisition and analysis as well as incompatibility with molecular phenotyping tools.

For light microscopy in large tissue samples, whole organs, or embryos, translucency is the dominant problem. While multi-photon microscopy allows tissue penetration up to $500 \mu \mathrm{m}$ without substantial light scattering, tissue clearing methods have rapidly evolved over the past decade. The advent of numerous tissue clearing methods ${ }^{4}$ has enabled unprecedented novel insight into the functional anatomy of numerous organs as well as whole embryos. ${ }^{6-10}$

*jwitzens@iph.rwth-aachen.de; phone +49 24180 20021; iph.rwth-aachen.de 
With tissue transparency, depth of focus is no more limited by the sample's optical properties, but rather dependent on the imaging setup, especially on the objective. Long working distance, high numerical aperture immersion objectives are required. Notably, samples are immersed in refractive index matching solution that differs from the standard refractive indices of water or oil. While optical sectioning is still required for post-hoc 3D reconstruction, point scanning techniques - even when using resonant scanners - are severely limited as data acquisition for larger samples at reasonable spatial resolution can easily take several days. Sample stability and focus drift are thus becoming serious issues for any experiment. Therefore, LSFM has become the standard method for imaging of cleared samples. ${ }^{6}$ LSFM substantially reduces both photobleaching and image acquisition time. ${ }^{11}$ When combined with a fast sCMOS camera, the camera's chip read-out time becomes the limiting factor for data acquisition speed. ${ }^{4}$

However, using LSFM comes with a significant speed-resolution trade-off. Axial light-sheet thickness (and, therefore, axial resolution), of course, depends on the configuration of the setup's optical components and the imaging light wavelength. With commercially available light-sheet microscopes tested using a $488 \mathrm{~nm}$ laser, axial resolution ranges between $1.7-15 \mu m$ (Leica TCS SP8 DLS), 2 - $14 \mu \mathrm{m}$ (Lightsheet Z.1; Zeiss), and 4.5 - $24 \mu \mathrm{m}$ (UltraMicroscope II; LaVision BioTec). At maximum fields of view in the $\mathrm{mm}$ range, commercial systems generate images that typically lack sub-cellular resolution.

In LSFM, a sample is illuminated with a thin sheet of light (xy plane) and imaged with an objective oriented in the perpendicular, axial (z-)direction. Spatial resolution in the plane of the light sheet (xy) is obtained, as in a conventional microscope, by the functionality of the imaging objective combined with imaging on a CMOS pixel array. Spatial resolution in the z-direction is obtained by a combination of the selective illumination (thickness of the light sheet) and the point spread function (PSF) of the recording objective, that is configured to have its focal point in the plane of the light sheet. This imaging technique is particularly useful to image large, cleared samples, due to its dramatically increased throughput compared to scanning-based optical sectioning methods (e.g., confocal microscopy).

Initial setups relied on generating a light sheet by rapidly scanning, along one axis $(\mathrm{x})$ of the light sheet, a Gaussian beam propagating along its other axis $(\mathrm{y})$. In this configuration, a limiting trade-off exists between the resolution in the axial z-direction, as determined by the Gaussian beam's field diameter $2 w_{0}$ at its focal point, and its depth of focus as given by twice its Rayleigh length $\pi w_{0}^{2} n / \lambda_{0}$, with $n$ the refractive index of the tissue and $\lambda_{0}$ the free space wavelength of the light $-702 \mathrm{~nm}$ in this work in order to excite the genetically encoded fluorescence protein miRFP720, ${ }^{12}$ chosen here for its compatibility with excitation at longer wavelengths. Thus, a beam diameter between $18 \mu \mathrm{m}$ (center of sheet) and 26 $\mu \mathrm{m}$ (edges of sheet) would be required to image a $1 \mathrm{~mm}$ sample depth (assuming the cleared sample to have the refractive index of water), very far from the diffraction limited spot-size limit and already at the upper limit for resolving individual neurons. Improving this trade-off, non-diffracting Bessel beams have been introduced into light sheet microscopy. ${ }^{13}$ These, however, retain two limitations: For one, only a Bessel beam of infinite extent in the xz plane (with y the direction of propagation) is truly non-diffracting. A truncated Bessel beam, as generated in a practical setup, keeps a finite diffraction length. Another important limitation resides in the side-lobes surrounding the center lobe of the beam: The radial dependency of the E-field strength of a Bessel beam scales as a Bessel function, resulting in a substantial amount of optical power contained in off-center side-lobes. This latter limitation in particular has been substantially improved by lattice light sheet illumination. Here, the concept of Bessel beam illumination is generalized to illumination with other non-diffracting beams, that now consist in a periodic, lattice-like illumination pattern bounded by an envelope function in the xz-plane. ${ }^{14}$ These lattice beams feature a spatially varying intensity profile along the $\mathrm{x}$-direction (the axis of the light sheet perpendicular to the direction of propagation) and can be used in one out of two configurations. Either, the lattice beam is rapidly dithered in the $\mathrm{x}$-direction, resulting in averaging during image capture, so that a uniform illumination in the $\mathrm{x}$ direction is emulated, but with suppressed or sufficiently spatially separated side-lobes in the z-direction to result in an improved PSF compared to Bessel beam illumination. Alternatively, the spatially varying profile in both the $\mathrm{x}$ - and $\mathrm{z}$ directions is used for structured illumination microscopy (SIM). A sequence of images is then taken, each time incrementing the position of the lattice beam in the x-direction by a corresponding fraction of the underlying lattice's periodicity, and the data further processed to obtain super-resolution imaging. Moreover, by extending the beam laterally along the width of the light sheet (in the x-direction), peak intensities can be substantially reduced while maintaining imaging speeds, thus reducing phototoxicity.

However, as for regular light sheet microscopy, a trade-off remains between the achievable resolution and the depth (along the direction of propagation) over which the beam can be used before residual diffraction leads to a significant degradation in the axial (z) resolution. In the following, we describe where this limitation arises from in state-of-the-art LSFM (Section II) and how we propose to remedy it with phased arrays implemented as silicon nitride photonic integrated circuits (Section III). 


\section{STATE-OF-THE-ART LATTICE LIGHT SHEET MICROSCOPY}

For both the generation of Bessel and lattice beams, a specific illumination pattern has to be generated at the rear pupil plane of a high numerical aperture objective that lies on a narrow annular aperture. Each point on this annular aperture is converted by the objective in a plane-wave with wave-vector $\left(k_{x}, k_{y}, k_{z}\right)$, with the k-vector component in the direction of propagation, $k_{y}$, equal for all the field components. As a consequence of the annular aperture, $\sqrt{k_{x}^{2}+k_{z}^{2}}-$ and thus also $k_{y}=\sqrt{k_{0}^{2}-k_{x}^{2}-k_{z}^{2}}$, with $k_{0}$ the magnitude of the k-vector - are equal for all the generated plane waves. Thus, all the field components share a common phase dependency in the y-direction, that can be factored out as ${ }^{14} E(r, t)=$ $\sum_{m} A_{m} e^{i\left(k_{x, m} x+k_{y} y+k_{z, m^{z}}-\omega t\right)}=e^{i\left(k_{y} y-\omega t\right)} \sum_{m} A_{m} e^{i\left(k_{x, m} x+k_{z, m} z\right)}$, with $A_{m}$ the amplitude of the component indexed by $m$, showing the translation invariance of the beam's power profile in the y-direction. Since the annular aperture necessarily has a finite width, the k-vector component $k_{y}$ also has a finite spread in practice and the beam eventually diffracts.

Previous lattice beam formation ${ }^{14}$ has been hindered by a number of factors: The beam formation has been obtained by combining a liquid-crystal based spatial phase modulator (SPM) operated in reflection mode, an annular aperture, two galvanometers to displace the beam in the x- and z-directions, and complex imaging optics. This costly and bulky setup would benefit from miniaturization, both to increase its stability and to reduce size and cost. Scanning speeds are limited by the actuation time of the SPM and of the galvanometers, which both have refresh rates in the order of a few $\mathrm{kHz}$. Improving the scan speed would be highly beneficial to reduce the de facto image acquisition time, which is required for real-time monitoring of fast signaling processes in living cells, and to reduce issues related to phototoxicity.

(a)

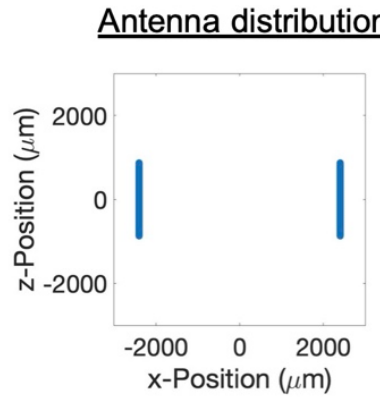

(b)

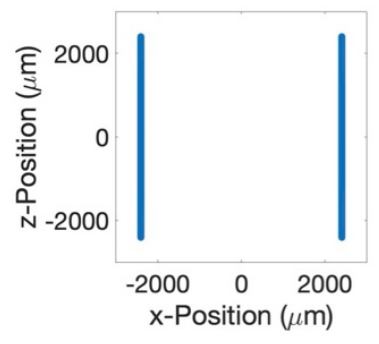

(c)

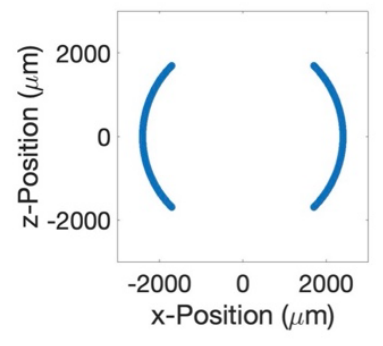

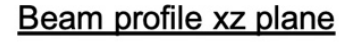
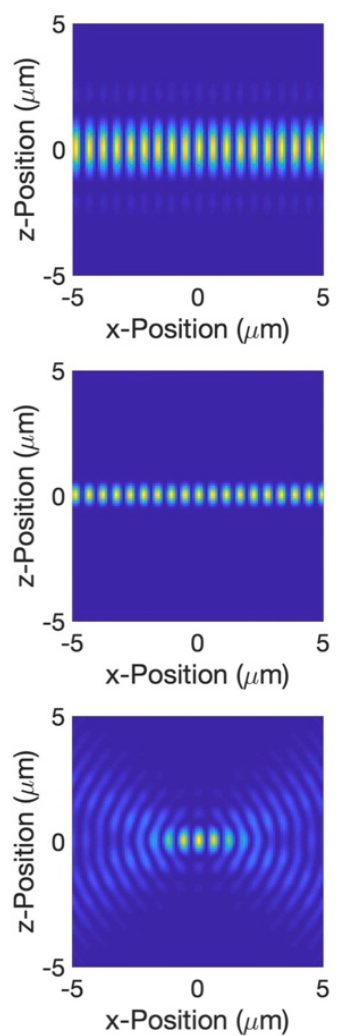

Beam profile along $z$
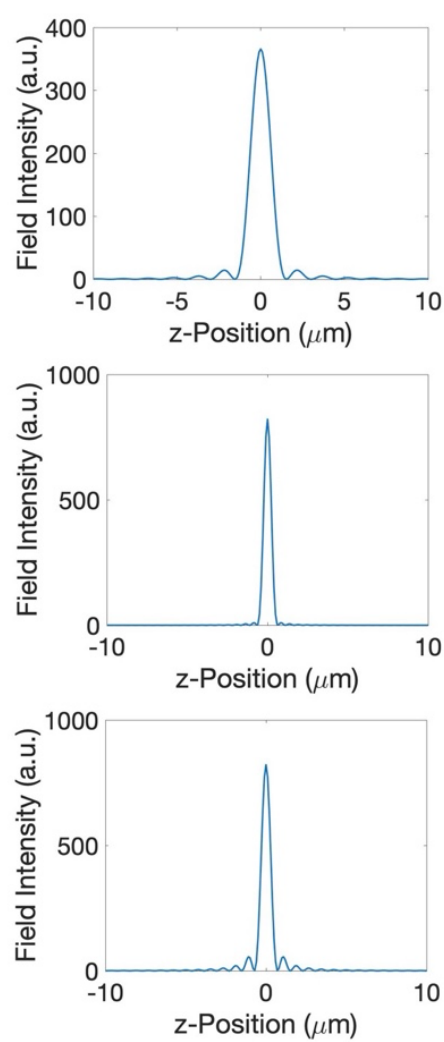

Beam profile yz plane
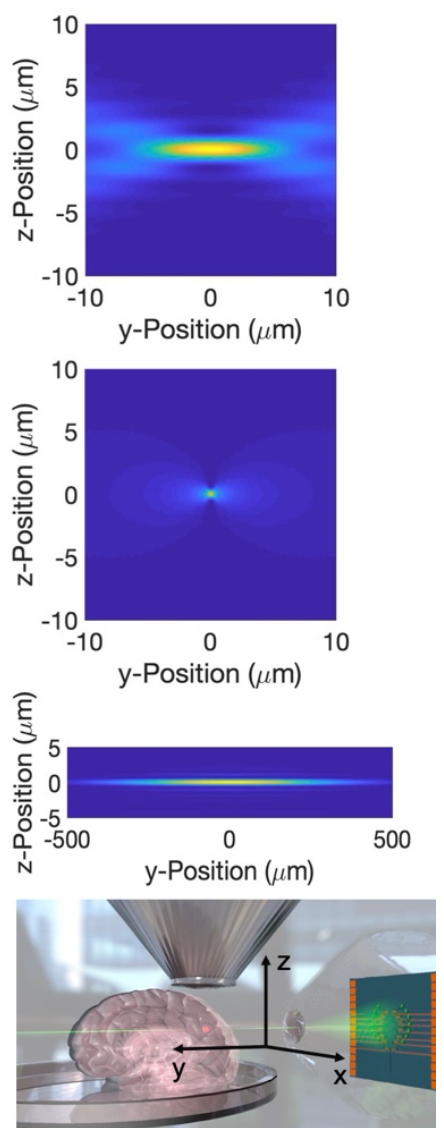

Figure 1. Exemplary reduced-diffraction beams generated by three different field / antenna distributions at the rear objective pupil (left column). The $2^{\text {nd }}$ and $4^{\text {th }}$ columns show the beam shape in the $x z$ plane (perpendicular to the direction of propagation) and in the yz plane (along the direction of propagation). The $3^{\text {rd }}$ column shows the field along the optical axis of the imaging objective (z-direction) at the center of the beam and determines the PSF. Axes are labelled in the 3D rendering shown in the lower right corner of the figure. (a) and (b) correspond to two exemplary lattice light sheets with different envelope functions in the z-direction. (c) corresponds to the field pattern generated by the proposed PIC. 
Beam diffraction and the achievable depth of the field of view (FOV), along the beam's direction of propagation, are currently limited by the width of the annular aperture used to spatially filter the field profile generated by the SPM. When operated as a pure phase sifter (as opposed to phase and amplitude modulation), an SPM is only imperfectly shaping the light, so that the use of an aperture is required to reduce side lobes at the rear pupil plane of the objective. This is particularly true when the SPM is operated in binary mode (with $180^{\circ}$ phase shifts) to increase refresh rates. This unavoidably also leads to substantial loss of optical power, made worse as narrow aperture widths and long diffraction lengths are targeted. Reducing the amount of lost light, together with a higher scanning speed, would facilitate the imaging of large tissue samples within reasonable time frames.

At a more fundamental level, beam diffraction and thus a restriction of the achievable FOV arises from the envelope function applied to the optical lattice. This is made apparent by comparing two lattice light sheets illustrated in Figs. 1(a) and 1(b). In both cases, the underlying 1D optical lattice is simply formed by two plane-waves with wave-vectors $\left( \pm k_{x, 0}, 0,0\right)$, resulting in an unbounded optical intensity distribution with periodicity $\Lambda=\pi / k_{x, 0}$. In order to bound the generated optical lattice in the z-direction, the two k-vectors are replaced by distributions $\left( \pm k_{x, 0}, 0, k_{z}\right)$, with $k_{z}$ bounded by $\left|k_{z}\right| \leq k_{z, \max }$. The larger $k_{z, \max }$, the tighter the confinement of the light in the z-direction and the better its respective PSF. However, increasing $k_{z \text {, max }}$ also leads to increased beam diffraction and thus to a reduction of the FOV. The larger the spread of the z-axis k-vector components $k_{z}$, the larger the departure from an ideal k-vector distribution along an annular aperture of zero width (Fig. 1, leftmost column), and the shorter the diffraction length along the y-axis (Fig. 1, rightmost column), however the more confined also the illumination pattern in the z-direction ( $3^{\text {rd }}$ column).

To exemplify this trade-off, we model beam formation with a high numerical aperture lens $(\mathrm{NA}=0.65,3.74 \mathrm{~mm}$ working distance ${ }^{14}$ ). The achievable resolution along the imaging axis (z) and the depth of the FOV depend on the height of the two field distributions at the rear objective pupil of respectively $1750 \mu \mathrm{m}$ and $4800 \mu \mathrm{m}$ (Figs. 1(a) and 1(b)), that map into the k-vector distributions after Fourier transformation by the objective. These result in center lobe full width at half maxima (FWHM) of $1.36 \mu \mathrm{m}$ and $0.54 \mu \mathrm{m}$, respectively. However, the reduced FWHM of configuration (b) comes at the cost of a much reduced field of view in the direction of the beam propagation (y) as seen in the $4^{\text {th }}$ column. While configuration (a) creates a light sheet over a depth of $\sim 5 \mu \mathrm{m}$, allowing imaging over a small cell, configuration (b) only allows imaging over a depth below a micron and can no longer be considered a "light sheet". In the following, we propose a solution to reach both sub-cellular resolution as well as a mm-scale FOV, overcoming this trade-off.

\section{LSFM WITH SILICON NITRIDE PHOTONIC INTEGRATED CIRCUITS}

Phased arrays are an alternate technology to generate arbitrary beam profiles ${ }^{15}$ Light is distributed on chip to an array of antennas, typically surface emitters implemented in the form of compact grating couplers, ${ }^{16}$ that emit light with a tunable phase in order to generate a targeted beam profile. The silicon nitride waveguide platform offers transparency at visible and short infrared wavelengths and is ideally suited to distribute and manipulate light in the wavelength range required for exciting fluorophores in the life sciences. ${ }^{17-19}$ Moreover, it has a very high damage threshold and vanishing two-photon absorption in the relevant wavelength range, so that it is not practically limited in the power it can handle. Recently, considerable progress has been made in silicon nitride phased arrays. ${ }^{20}$ In particular, one of the difficulties that has been associated to phased arrays is the so-called coherence length of the waveguides. ${ }^{21}$ Due to manufacturing induced variations in waveguide widths and ill-controlled layer thicknesses or side-wall roughness, the optical path length of waveguides shows small deviations from their design target, that can vary from waveguide to waveguide and chip to chip. In order to correct for these, phase tuners need not only to apply the phase required to generate a given field profile, as can be predictively determined with an algorithm, but also need to correct for these fabrication induced phase shifts. This can be a harder problem as the phase errors are not a-priori known and need to be determined from an initial chip characterization or an adaptive optimization. State-of-the-art fabrication tolerances have however undergone sufficient progress so that the coherence length of silicon nitride may be long enough to implement scaled phased arrays without requiring correction. ${ }^{20,21}$ This is analyzed in more details below for the specific photonic integrated circuit (PIC) architecture considered here.

We model beam formation with a phased array implemented on a 3 by $3 \mathrm{~mm}$ PIC and the high numerical aperture lens $(\mathrm{NA}=0.65,3.74 \mathrm{~mm})$ already assumed in the previous section. Individual antennas are conservatively assumed to emit Gaussian beams with a $1 / \mathrm{e}^{2}$ waist diameter of $6 \mu \mathrm{m}$ and are spaced by $7.5 \mu \mathrm{m}$ from each other along the contours shown in the left column of Fig. 1, close enough to avoid the formation of higher order artifacts in the formed beams. Gaussian beams are propagated to the image plane of the lens using the ABCD matrix method and the resulting interferometric patterns computed. Individual Gaussian beams cover a disk with a diameter of $460 \mu \mathrm{m}$ after imaging $(25 \%$ intensity at the edge relative to center), giving a rough indication of the area in the xz-plane in which beams can be scanned with the 
phased array (as given by the form factor of the diffractive pattern). The two shaped illumination beams already discussed in the previous section, as shown in Figs. 1(a) and 1(b), can also be generated with a PIC (simulated with respectively 512 and 1024 antenna elements).

In order to overcome the trade-off between sub-cellular resolution and extended FOVs, we introduce a third antenna distribution as shown in Fig. 1(c), also comprising 1024 antenna elements. In this case, the antenna elements are forced again to lie on a circle, resulting in a near diffraction-less beam over a propagation length of $1 \mathrm{~mm}$ only limited by the size of the individual antenna elements, causing the only deviation from an ideal circular field emitter distribution. Concomitantly, a FWHM of $0.66 \mu \mathrm{m}$ is maintained in the z-direction.
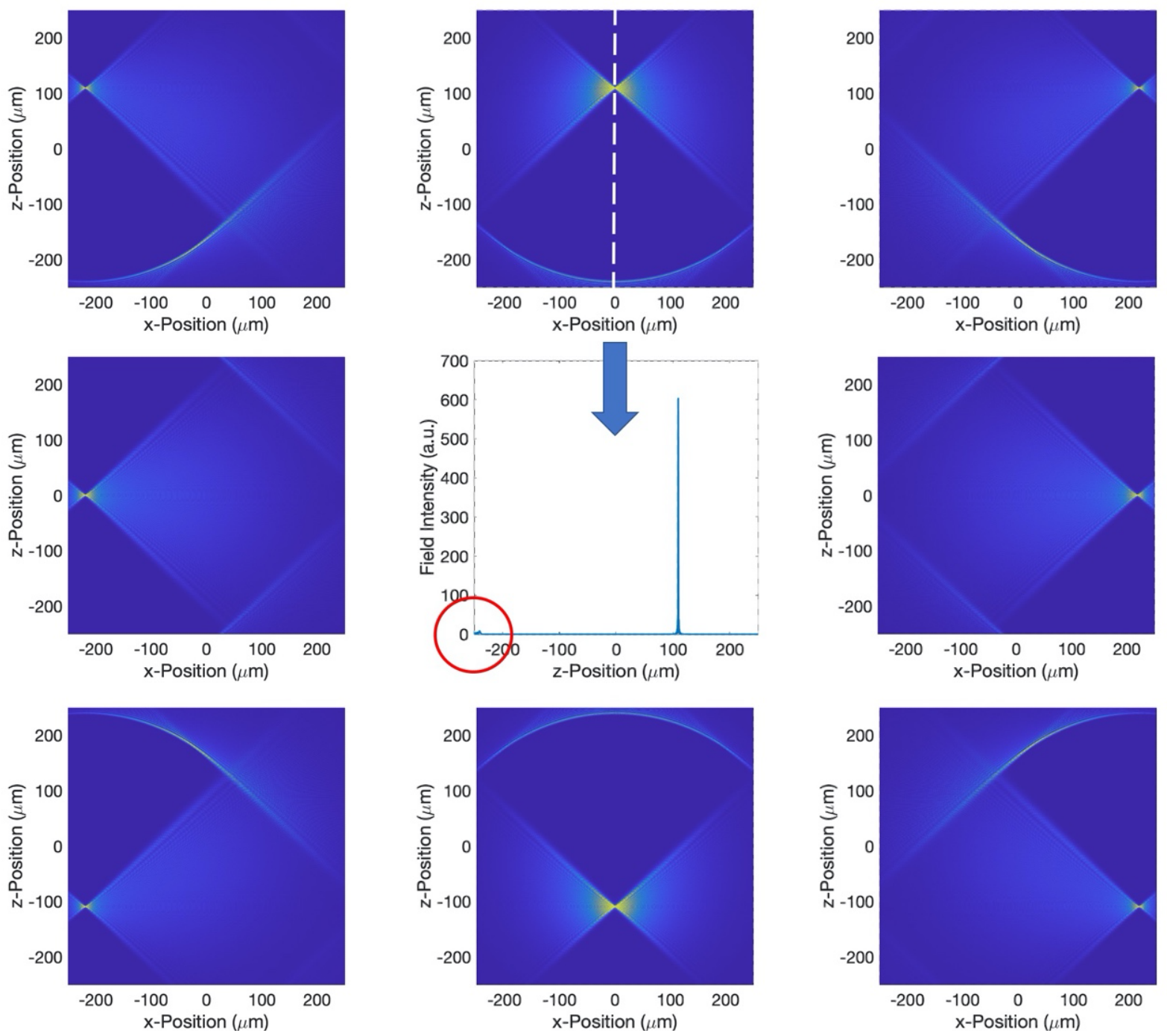

Figure 2. Scanning of the lattice light sheet in the $\mathrm{x}$ - and $\mathrm{z}$-directions. The central plot shows the intensity distribution along the $\mathrm{z}-$ direction, across the center of the beam, when it is displaced by $100 \mu \mathrm{m}$ in the z-direction (dashed white axis in the top graph). While the formation of a small artefact is visible at $\mathrm{z}=-240 \mu \mathrm{m}$, its integrated intensity is $10 \mathrm{X}$ below that of the main beam and its position way out of the focal plane of the imaging objective.

However, a second trade-off now comes into play: While for the initial, unbounded optical lattice described in Section II only two x-axis k-vector components $\pm k_{x, 0}$ came into play, each is now transformed into a distribution, so that the perfect periodicity of the resulting lattice is broken in the x-direction. As seen in the second column of Fig. 1(c), the lattice now only keeps its shape in a range of $\pm 1.5 \mu \mathrm{m}$ along the $\mathrm{x}$-direction ( $\sim 5$ lobes), after which it rapidly loses confinement in the z-direction. In other words, we sacrificed extension of the optical lattice in the x-direction in order to gain extension along the direction of propagation (y). However, since the PSF in the x-direction is determined by the imaging system and 
the lattice beam can be translated in the x-direction, this does not fundamentally limit the FOV of the microscope. Rather, pixels have to be recorded sequentially as the lattice beam position is scanned in the $\mathrm{x}$-direction. Since silicon nitride thermal phase tuners have been shown to be switchable in the double digit $\mathrm{kHz}$ range ${ }^{22}$ and ongoing work on ferro-electric material integration might enable even higher scanning speeds, ${ }^{23}$ we may reach a regime in which the camera readout time remains the only limiting factor. Moreover, in the following we will show a straightforward scheme to implement the beam steering based on scanning of the laser wavelength.

Scanning in the $\mathrm{x}$ - and $\mathrm{z}$-directions is obtained by incrementing the phase of antenna elements according to their $\mathrm{x}$ - and z-ordinates, respectively. Modeling results are shown in Fig. 2. It is apparent that the beam can be scanned $\pm 200 \mu \mathrm{m}$ in the $\mathrm{x}$-direction and $\pm 100 \mu \mathrm{m}$ in the z-direction with minimal artefact formation, with a peak intensity $70 \mathrm{X}$ below the main beam (the maximum of the color scale was scaled down to make this artefact apparent) and an intensity integrated over the $\mathrm{z}$-axis 10X below that of the main beam. Moreover, the position of the artefact is way out of the focal plane of the imaging objective. Thus a total volume of $400 \mu \mathrm{m} \times 1 \mathrm{~mm} \times 200 \mu \mathrm{m}$ is addressable without moving the sample or the lens (although scanning in the z-direction is of reduced utility as imaging is also limited by the focal plane of the imaging objective, it may serve to fine align the illumination pattern with the latter or to support SIM). Thus, the light sheet can cover a $400 \mu \mathrm{m}$ by $1 \mathrm{~mm}$ area in the xy-plane while maintaining its high inherent resolution.
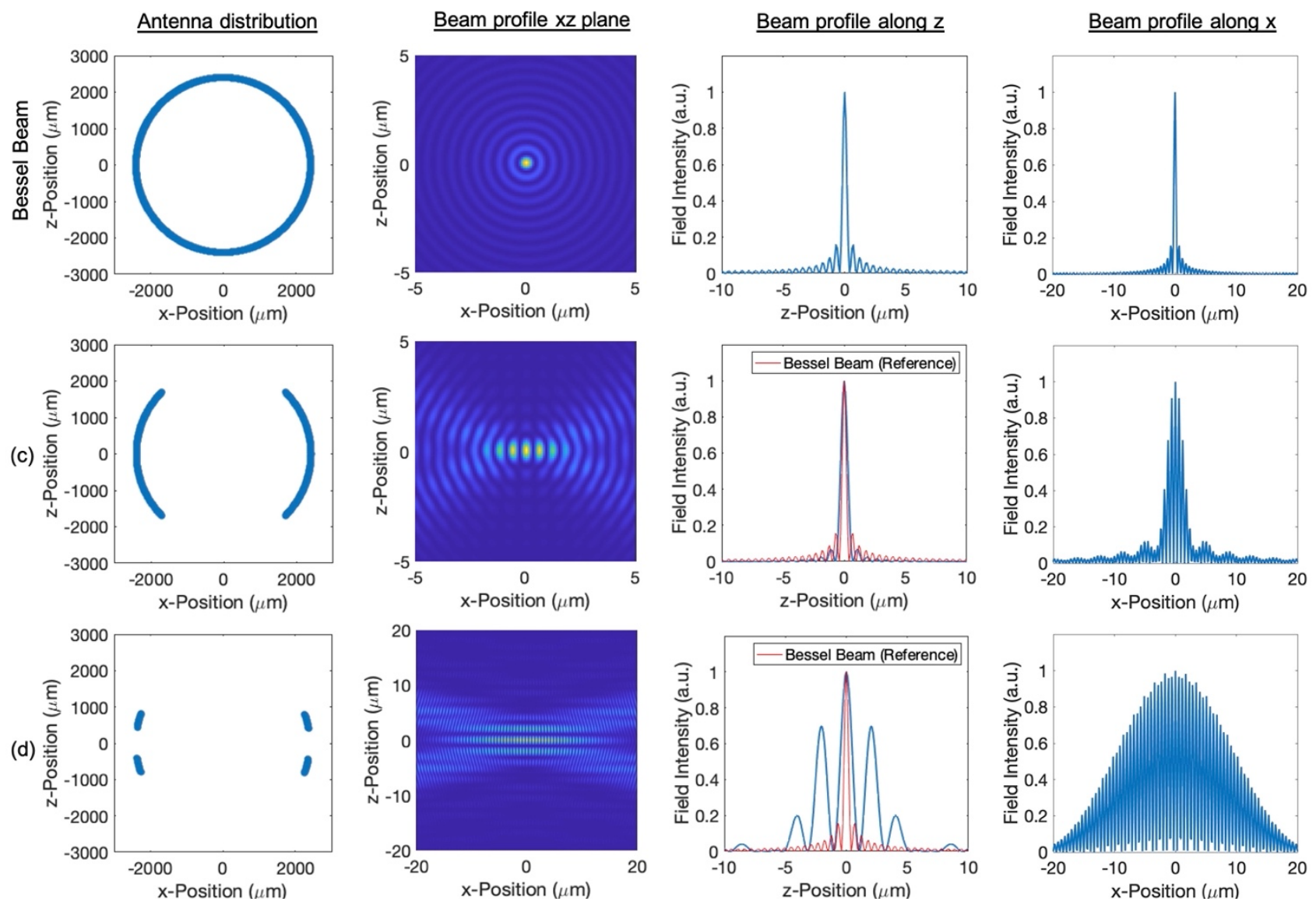

Figure 3. Comparison between a Bessel beam, antenna configuration (c), identical to the one shown in Fig. 1(c), and a further configuration (d) resulting in a 2D lattice-like illumination at the center of the beam without sacrificing the suppressed beam diffraction. The beam is significantly expanded in the lateral $x$-direction at the price of a modest overall imaging PSF increase compared to (c). In the $\mathrm{z}$-axis beam profiles, the Bessel beam profile is overlaid in red as a common reference.

Lattice light sheet microscopy as shown by Eric Betzig and colleagues ${ }^{14}$ keeps a significant advantage over the configuration shown in Fig. 1(c), which is to provide a laterally extended light sheet (along the x-direction) resulting in much reduced peak intensities and phototoxicity. The limited lateral extent of the beam generated by configuration (c), while significantly larger than that of a Bessel beam of comparable illumination PSF (Fig. 3), is still only a few $\mu \mathrm{m}$. This can be improved on by further applying the concepts reported in ref. 14 to the circular antenna distribution used here. Fig. 
3 compares the lateral (x) and vertical (z) beam profiles of a Bessel beam, the beam generated by the antenna distribution (c) that in its center region corresponds to a bounded 1D lattice, and a third beam corresponding to a bounded 2D lattice generated by a further antenna distribution (d). The extent of the beam (d) is much larger in the z-direction, as a consequence of the truncation of the antenna distribution in the z-direction (antennas are restricted to an angle below $20^{\circ}$ relative to the $\mathrm{x}$-axis, as opposed to $45^{\circ}$ in distribution (c)). Since this truncation reduces the spread of the antenna distribution in the $\mathrm{x}$-direction even more, the lattice is significantly broadened in the $\mathrm{x}$-direction and reaches a lateral FWHM of $20 \mu \mathrm{m}$, allowing a substantial reduction in peak illumination intensities. In the z-direction, beam (d) features multiple lobes resulting from the 2D lattice structure, with the central lobe featuring a FWHM of $1 \mu \mathrm{m}$ slightly larger than that of configuration (c). Since the other lobes are out of focus, the overall PSF is largely dominated by this central lobe. As a consequence, the imaging depth in the beam's propagation direction $(y)$ remains unchanged, while the power is spread over a substantial width in the lateral $\mathrm{x}$-direction at a modest price in terms of the imaging PSF.

A diagram of the envisioned PIC is shown as Fig. 4. Light is distributed to the antenna elements with a tree network exemplarily based on one by two multi-mode interferometers (MMIs). It is first split between the two sides of the phased array by a first MMI, after which 9 layers of MMIs split the light on each side between 512 antenna elements (three layers are shown in Fig. 4 for illustration purposes). The optical path lengths are all balanced by design up to the outputs of the last MMI layer, with equal numbers of bends, straight segments on a cartesian grid and MMIs. The last segments connecting the outputs of the last MMI layer to the antennas are of different lengths in order to reach the circumference of the circle defining the locus of the antennas (dashed line in Fig. 4). This unbalancing is purposefully introduced and will serve for horizontal beam steering (beam displacement along the x-axis) in the following. The position of individual antenna elements can be slightly shifted along the $\mathrm{x}$-axis, in order to ensure a common phase is obtained for all at the target wavelength at the center of the fluorophore's absorption spectrum (in our case, $702 \mathrm{~nm}$ for miRFP720). While this requires knowledge of the interconnect waveguide effective index $n_{e f f}$, an error $\delta n_{\text {eff }}$ below \pm 0.01 could for example be corrected by shifting the laser wavelength by $\pm 3.5 \mathrm{~nm}$ with small impact on the protein's absorption cross-section.

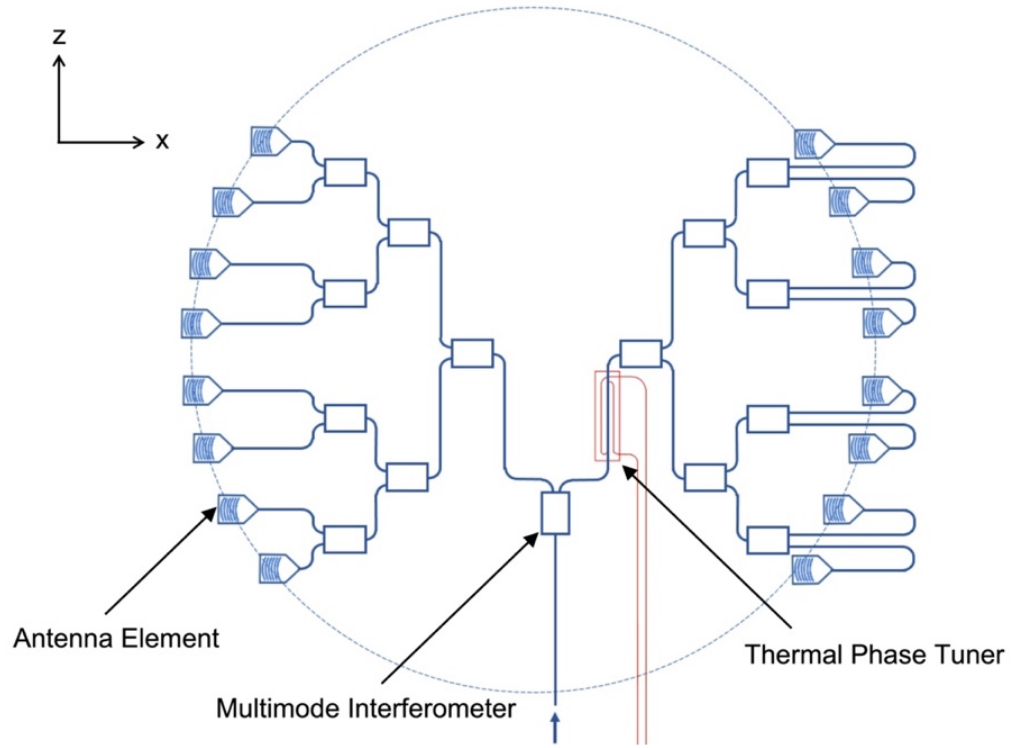

Figure 4. Diagram of the envisioned PIC. After splitting the light between the left and right side of the PIC with a first MMI, light distribution trees with nine layers of cascaded MMIs split the light among 512 antenna elements on each side. A thermal phase tuner allows adjusting the relative phase between the two sides of the PIC, for rapid dithering of the optical lattice lobe positions. Displacements along the x-axis of the whole beam (envelope function) is obtained by changing the laser wavelength.

The path length between the first MMI at the beginning of the light distribution tree on either side of the PIC and the antenna elements is below $4 \mathrm{~mm}$. Such path lengths have previously been measured to result in a phase error variance on the order of $0.3 \mathrm{rad}^{2}$ if fabricated in $300 \mathrm{~mm}$ technology and $1.5 \mathrm{rad}^{2}$ if fabricated in $200 \mathrm{~mm}$ technology. ${ }^{21}$ Thus, the PIC should work without further correction if fabricated in $300 \mathrm{~mm}$ technology, while the consequences of phase errors in 200 $\mathrm{mm}$ technology warrant further investigation. Monte-Carlo simulations of the PIC show however that the performance penalties due to the increased phase error in $200 \mathrm{~mm}$ technology should also remain acceptable without further trimming. The power profile of the generated beam remains very close to the one generated in the absence of phase errors, but with 
an overall reduction due to a portion of the power being spread over the sample as a consequence of the random phase errors. While assuming fully uncorrelated phase errors for each antenna element with a variance of $1.5 \mathrm{rad}^{2}$ results in only $\sim 20 \%$ of the power remaining inside the beam, taking into account correlations in the phase errors resulting from shared optical paths in the physical layout leads to $\sim 80 \%$ of the power remaining in the central lobe (with a $\pm 20 \%$ confidence interval as given by one std. dev.).

A phase tuner allows adjusting the relative phase between the two sides of the PIC. This shifts the position of the lobes of the underlying optical lattice, i.e., the position of the lobes shown in the second column of Figs. 1(c) and 3(d). This can be used for rapid dithering, either to average the lobes or for recording successive images when using structured illumination. However, the envelope function of the beam, i.e., the x-position at which the narrow PSF is obtained, is not shifted. The whole beam envelope function can be modified by changing the wavelength, as this will modify the relative phase between the antenna elements according to their $\mathrm{x}$-axis position.

The sensitivity on wavelength of the optical phase at a given antenna element at position $x$ is given by $\Delta \varphi=$ $-2 \pi n_{g} x \Delta \lambda_{0} / \lambda_{0}^{2}$, where $n_{g}$ is the group index. The objective in the illumination path also converts the illumination pattern of a point source at position $x$ into a plane-wave with $\mathrm{x}$-axis k-vector component $k_{x}=k_{0} \cdot \sin (\operatorname{atan}(x / W D)) \simeq k_{0}$. $x / W D$, with $W D$ the working distance of the objective. The lateral $\mathrm{x}$-axis displacement of the beam is thus given by $\Delta x=$ $\partial \varphi / \partial k_{x}=-W D \cdot\left(n_{g} / n_{e f f}\right) \cdot(\Delta \lambda / \lambda)$. Given the $3.74 \mathrm{~mm}$ working distance assumed here, a $\pm 50 \mu \mathrm{m}$-axis displacement would be achieved with a wavelength shift of $\pm 10 \mathrm{~nm}$ remaining in the high absorption range of the miRFP720 fluorescent protein ( $>85 \%$ absorption efficiency compared to maximum at $702 \mathrm{~nm}$ ). The full $\pm 200 \mu \mathrm{m}$ beam steering shown in Fig. 2 would be achievable with the same wavelength range assuming additional delay loops are introduced in the last segments of the light distribution trees, wherein the length of these delay loops are sized according to the $\mathrm{x}$-axis position of the antenna elements, enhancing the corresponding wavelength dependency of the emission phase. We are currently also working on the development of suitable wavelength tunable external cavity lasers combining reflective semiconductor optical amplifiers with silicon nitride PICs.

\section{CONCLUSIONS}

In conclusion, generating lattice light sheets with silicon nitride photonic integrated circuits would not only enable lower cost, more robust, more compact, and more power efficient microscopy solutions, but also increase the diffraction limited depth of the field of view, only limited by the size of the antenna elements placed on a perfect circular distribution, while concomitantly maintaining sub-cellular resolution. While this comes at the cost of a reduced useable range in the light sheet direction perpendicular to the beam's direction of propagation, this can be remedied by rapid scanning of the beam along this in-plane direction with a straightforward PIC configuration. A compromise has to be struck between the axial resolution and the lateral extent of the beam, with the latter an essential parameter to reduce illumination intensities and thus phototoxicity. We expect sub-cellular imaging resolutions to be compatible with millimeter sized fields-of-view along the propagation axis of the illumination beam with this illumination scheme.

\section{REFERENCES}

[1] Holekamp, T. F., Turaga, D. and Holy, T. E., "Fast three-dimensional fluorescence imaging of activity in neural populations by objective-coupled planar illumination microscopy," Neuron 57, 661-72 (2008).

[2] Ahrens, M. B., Orger, M. B., Robson, D. N., Li, J. M. and Keller, P. J., "Whole-brain functional imaging at cellular resolution using light-sheet microscopy," Nat. Methods 10, 413-420 (2013).

[3] Keller, P. J., Ahrens, M. B. and Freeman, J., "Light-sheet imaging for systems neuroscience," Nat. Methods 12, 2729 (2014).

[4] Richardson, D. S. and Lichtman, J. W., "Clarifying Tissue Clearing,” Cell 162, 246-257 (2015).

[5] Denk, W., Briggman, K. L. and Helmstaedter, M., "Structural neurobiology: missing link to a mechanistic understanding of neural computation," Nat. Rev. Neurosci. 13, 351-358 (2012).

[6] Chung, K. and Deisseroth, K., "CLARITY for mapping the nervous system," Nat. Methods 10, 508-13 (2013).

[7] Yang, B. et al., "Single-Cell Phenotyping within Transparent Intact Tissue through Whole-Body Clearing," Cell 158, 945-958 (2014).

[8] Chung, K. et al., "Structural and molecular interrogation of intact biological systems," Nature 497, 332-337 (2013).

[9] Renier, N. et al., "iDISCO: A Simple, Rapid Method to Immunolabel Large Tissue Samples for Volume Imaging," Cell 159, 896-910 (2014). 
[10] Susaki, E. A. et al., "Whole-Brain Imaging with Single-Cell Resolution Using Chemical Cocktails and Computational Analysis," Cell 157, 726-739 (2014).

[11] Conchello, J.-A. and Lichtman, J. W., “Optical sectioning microscopy,” Nat. Methods 2, 920-931 (2005).

[12] Matlashov, M. E. et al., "A set of monomeric near-infrared fluorescent proteins for multicolor imaging across scales," Nat. Commun. 11, 239 (2020).

[13] Planchon, T. A. et al., "Rapid three-dimensional isotropic imaging of living cells using Bessel beam plane illumination," Nat. Meth. 8(5), 417-423 (2011).

[14] Chen, B.-C. et al., "Lattice light-sheet microscopy: Imaging molecules to embryos at high spatiotemporal resolution," Science 346(6208), 1257998 (2014).

[15] Sun, J. et al., "Large-Scale Silicon Photonic Circuits for Optical Phased Arrays," J. Sel. Top. Quant. Electron. 20(4), 8201115 (2014).

[16] Romero-García, S. et al., "Visible wavelength silicon nitride focusing grating coupler with AlCu/TiN reflector," Opt. Lett. 38(14), 2521-2523 (2013).

[17] Romero-García, S. et al., "Silicon nitride CMOS-compatible platform for integrated photonics applications at visible wavelengths," Opt. Expr. 21(12), 14036-14046 (2013).

[18] Mashayekh, A. T., Klos, T., Koch, S., Merget, F., Geuzebroek, D., Klein, E., Veenstra, T., Büscher, M., Leisching, P. and Witzens, J., "Miniaturized PIC multi-color laser engines for the life sciences," Proc. SPIE 10922, 109221U (2019).

[19] Mashayekh, A. T., Klos, T., Geuzebroek, D., Klein, E., Veenstra, T., Büscher, M., Merget, F., Leisching, P. and Witzens, J., "Silicon nitride PIC-based multi-color laser engines for life science applications," Opt. Expr. 29, (2021).

[20] Poulton, C. V. et al., "Large-scale silicon nitride nanophotonic phased arrays at infrared and visible wavelengths," Opt. Lett. 42(1), 21-24 (2017).

[21] Saseendran, S. S. et al., "A 300mm CMOS-compatible PECVD silicon nitride platform for integrated photonics with low loss and low process induced phase variation," Proc. Opt. Fib. Conf. (OFC), M1C.4 (2019).

[22] Mohanty et al., "Reconfigurable nanophotonic silicon probes for sub-millisecond deep-brain optical stimulation," Nat. Biomed. Eng. 4, 223-231 (2020).

[23] Alexander, K. et al., "Nanophotonic Pockels modulator on a silicon nitride platform," Nat. Comm. 9, 3444 (2018). 